\title{
Familial progressive supranuclear palsy
}

\author{
Jeremy Brown, Peter Lantos, Michael Stratton, Penelope Roques, Martin Rossor
}

\begin{abstract}
A progressive extrapyramidal syndrome and dementia occurred in three members of one family. The age of onset was in the seventh decade and the affected individuals showed many of the clinical features of progressive supranuclear palsy (PSP). Necropsy of one individual revealed the neuropathological features of PSP. We propose that this family has a familial form of PSP and review the evidence in the literature that a familial form exists.
\end{abstract}

(F Neurol Neurosurg Psychiatry 1993;56:473-476)

Progressive supranuclear palsy (PSP) was first defined as a clinical and pathological syndrome by Steele et al in 1964..$^{1}$ They described a series of nine patients with a combination of a supranuclear gaze palsy, dementia, axial and limb rigidity, and pseudobulbar palsy. Seven of the nine were studied pathologically and showed neuronal loss, granulovacuolar degeneration, gliosis, and neurofibrillary tangles affecting mainly subcortical structures. They recognised some variation in the clinical presentation of their patients. In the majority the dementia was mild, but three patients with a more severe dementia were included in the series because they had oculomotor and neuropathological abnormalities similar to the other cases. Psychometric studies in PSP reveal a subcortical dementia ${ }^{2}$ with evidence of frontal lobe dysfunction. ${ }^{3}$ Positron emission tomography shows a frontal deficit in both cerebral blood flow and glucose utilisation studies. ${ }^{45}$

We describe the clinical features of three members of one family who developed dementia with extrapyramidal features in their sixties. One individual had a post mortem examination which showed the pathological appearances of progressive supranuclear palsy. We discuss the diagnosis in this family and review other cases of possible familial PSP.

\section{Methods}

This family contacted us as a result of our studies into familial Alzheimer's disease. Two members of the family, the proband (3.5) and her cousin (3.1), were seen by us. The proband had a post mortem examination. The pedigree is shown in fig 1 .
INDIVIDUAL 3.5

The proband presented with a depressive illness at age 62 years. She was started on lofepramine $70 \mathrm{mg}$ per day. Two years later she developed an unsteady gait, tended to veer to the left, and had several falls. Her memory began to deteriorate and she complained of episodes of transient difficulty in speaking. On examination the only abnormalities found were poor coordination of the left hand and absent ankle jerks. Neuropsychological testing showed a verbal IQ of 91 and performance of 86 (WAIS-R); her estimated premorbid IQ was 112 (Schonell graded word test) and she had difficulties with visuospatial tasks. Specific tests of frontal lobe function were not done. A brain CT scan showed moderate enlargement of the ventricles with generalised atrophy with slight speckled calcification in both corpora striata. She was started on aspirin.

Over the next six months she complained of intermittent double vision, pressure behind her eyes, insomnia, and dysphagia particularly for solids. She retained some insight into her illness. When seen by one of us (PR) one year later she walked with a Zimmer frame and needed help with all activities of daily living. She had a tremor of the left hand and increased tone in all limbs. She spoke extremely slowly. Her Mini-Mental test ${ }^{6}$ score was 23. Her disabilities fluctuated. Over the next year she deteriorated until she died aged 71. Necropsy was performed and neuropathological findings are described below.

\section{INDIVIDUAL $2 \cdot 4$}

The mother of the proband presented with depression and personality change aged 69 years. She became unsure of her balance and worried that young children might knock her over. Her hands became clumsy and painful. A diagnosis of Parkinson's disease was suggested but she had no treatment. She became aggressive and cruel in her conversation. Over the next two years she developed severe dysarthria, became wheelchair bound, and, at the age of 72 years, was confined to bed. Her memory deteriorated, although her cognitive ability was variable. She died aged 73 years.

INDIVIDUAL $3 \cdot 1$

A cousin of the proband, a precise and meticulous person, worked as a bank supervisor. At age 61 years her handwriting deteriorated. She became careless over her appearance, unhygienic, and forgetful. She 




Figure 1 Family pedigree. Affected family members are shown with shaded symbols. Ages of death are shown below the symbols.

became obsessive and bought 100 pairs of trousers and 200 blouses from the same shop in a few weeks. She stopped washing clothes and filled the upstairs rooms of her house with dirty garments. Her mood was usually euphoric but she could be verbally and physically aggressive to her husband and made sexual suggestions to young relatives. Her speech became slurred. At age 65 years a CT scan showed mild cerebral atrophy without other abnormalities, but an EEG was normal. She was started on haloperidol.

Her deterioration continued; she became noisy and aggressive and she developed urinary incontinence. The haloperidol was stopped. She began eating excessively and gained weight. She developed pemphigus which was treated with low doses of steroids. Aged 68 she started constant mouthing of objects, chewing a pillow or sucking her hand. She spoke rarely but understood simple commands. She remained aggressive trying to bite staff. She was not treated with levodopa.

When seen by us aged 69 years she used single words occasionally but correctly. When choosing food she would say "fish" or "salad" and could give her date of birth when asked. She cooperated with commands but had difficulty in initiating actions and needed help with eating and washing. The general medical examination was unremarkable. She had orofacial dyskinesia and dribbled from the mouth. She had a supranuclear gaze palsy with restriction of voluntary gaze in all directions; lateral pursuit was broken. On completion of gaze horizontally and vertically there was coarse nystagmus which fatigued rapidly. Convergence of gaze was restricted. The jaw jerk was exaggerated. Voluntary movements of the palate were normal, gag reflex was preserved, and she drank a glass of water without difficulty. Tongue movements were slow. She had no orofacial dyspraxia. There was an increase in axial and limb tone with no tremor or fasciculation. She had marked bradykinesia without weakness and tendon reflexes were normal. The right plantar response was flexor, the left plantar extensor.
Limb coordination was normal and sensation intact. Her gait was apraxic, wide based with small steps, and she walked with a marked stoop owing to a forward bend of her lumbar spine. There was a prominent rooting reflex to visual and tactile stimuli, a pout reflex, and bilateral grasp reflexes. Her blink rate was reduced.

All tasks were performed extremely slowly and she was easily distracted. She could communicate using a point chart with help in initiating her hand movement. She was fully orientated and knew her date of birth. She completed a two stage task choosing a bar of soap from four items and then placing it behind a photograph. She performed simple calculations with some errors which she corrected on repetition. When asked to name an animal which was large and grey with a trunk, she pointed to the word elephant. However, she pointed to the previous prime minister (Margaret Thatcher) when asked to name the current one and did not pick a stool as an object with no back to sit on. Initially she pointed to the correct names of nine pictures in the Queen Square Naming Test, but scored less well on repetition. She had marked micrographia and her handwriting was illegible.

\section{UNAFFECTED INDIVIDUALS}

Individuals $1 \cdot 1$ and $1 \cdot 2$ died aged 71 and 62 respectively; 1.2 had heart disease. Ages of death for other individuals are shown in figure 1. Individual 3.6 had cognitive problems thought to be due to a cerebral arteriovenous malformation. Several individuals in the third generation are below the mean age of onset of the disease, one of these had an episode of myelitis but made a complete recovery. There were no other neurological or psychiatric disorders in the family.

\section{NEUROPATHOLOGY ON INDIVIDUAL 3.5}

The fixed brain weight was $1070 \mathrm{~g}$ and the brainstem and cerebellum weighed $165 \mathrm{~g}$. The leptomeninges were slightly thickened. The cranial nerves were normal. The large cerebral arteries showed many atherosclerotic plaques with narrowing of the lumina up to $25 \%$. On coronal slicing the lateral ventricles and the third ventricle were enlarged and the cerebral aqueduct was slightly dilated. The Sylvian fissures and many sulci were widened, and there was additional space between the hippocampus and the wall of the temporal horn of the lateral ventricles. The substantia nigra was somewhat pale but the locus coeruleus appeared well pigmented. No focal abnormalities were seen.

Blocks of tissue were taken from the frontal, temporal, parietal and occipital lobes, basal ganglia, amygdala, thalamus, cerebellar vermis and hemisphere, midbrain, pons, and medulla oblongata. Altogether 23 blocks were sampled. Sections were stained with haematoxylin and eosin, luxol fast blue, and cresyl violet and impregnated with silver according to Marsland and Glees, and Gallyas. Immunocytochemistry was carried out using 
the avidin biotin complex (Amersham, UK) with the following antibodies: BF10 (a monoclonal antibody to the phosphorylated medium weight polypeptide of neurofilaments), RT-97 and 147, (both monoclonal antibodies to the phosphorylated heavy weight neurofilament polypeptide), tau and BA4 (12-28) (all kindly provided by Professor BH Anderton, Institute of Psychiatry, London), ubiquitin and GFAP (both Dako, UK).

On histology, the major finding was of neurofibrillary tangles, some flame shaped, some globose, in the locus coeruleus, pontine nuclei, substantia nigra, inferior olivary nuclei, dentate nucleus, arcuate nucleus, raphe nuclei, globus pallidus, and nucleus basalis of Meynert. There were only very few tangles in the amygdala, hippocampus and parahippocampal gyri, or in the neocortical areas. Quantitative assessment was not carried out, but in the locus coeruleus, which was symmetrically affected, about $50 \%$ of the neurons contained tangles. A few plaques were seen only in subpial zone of the amygdala and the BA4 deposits were few and small. Granulovacuoles were seen in the substantia nigra and pontine nuclei, but not in the cortical areas. Silver impregnation according to Gallyas revealed many neuropil threads in the cortex. Very few cells remained in the dentate nucleus and the superior cerebellar peduncle was pale. Grumose change was seen around many dead or dying dentate nerve cells. The olivary nuclei were not, however, enlarged, nor was there evidence of extensive arborisation of dendrites or nerve cell hypertrophy. There was extraneuronal pigment in the substantia nigra and locus coeruleus. The hippocampus showed a tiny old infarct.

The neurofibrillary tangles gave a strongly positive reaction with antibodies to tau protein (fig 2), but were negative or weakly positive with ubiquitin, and were entirely negative with antibodies BF10, RT97, and 147. There was patchy, moderate astrocytosis, demonstrated by GFAP, in many areas of the deep grey matter.

The pattern of distribution of tangles in the

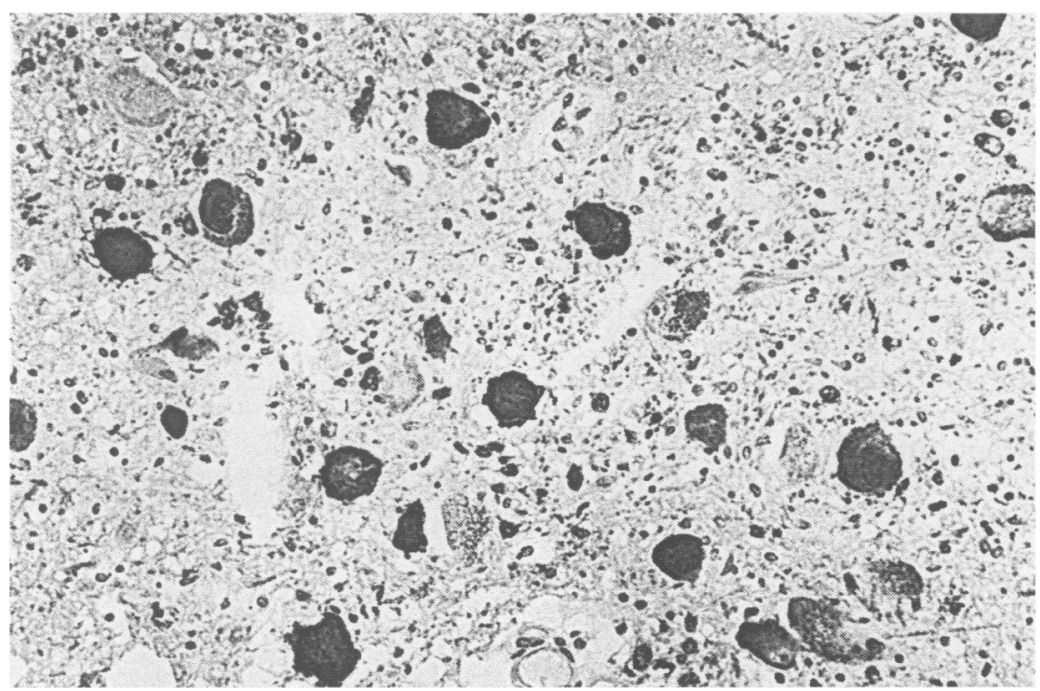

Figure 2 Neurofibrillary tangles in the locus coeruleus immunostained with an antibody to tau protein. Avidin-biotin complex reaction. $\times 200$. absence of plaques is most consistent with progressive supranuclear palsy. The striking paucity of tangles and plaques in the hippocampus is against the diagnosis of Alzheimer's disease.

\section{Discussion}

Three members of this family developed a dementia, extrapyramidal syndrome, gait disturbance, and dysarthria in their sixties. This combination of symptoms and signs is very unusual and is unlikely to have occurred by chance; they are the commonest presenting features in PSP. ${ }^{7}$ The patient examined by a neurologist would fulfil clinical criteria for the diagnosis of $\mathrm{PSP}^{8}$ were it not for her positive family history. One individual had a neuropathological examination which showed the changes of PSP. The involvement of two generations and a cousin makes a common environmental factor unlikely and suggests this disease is a genetic one; the mode of inheritance is compatible with an autosomal dominant gene. Therefore we suggest that these individuals have a familial form of PSP. There is some variation in the features between the family members but this is well within the spectrum reported in other autosomal dominant dementias such as familial prion disease. ${ }^{9}$

The majority of reported cases of PSP have demonstrated a consistent clinical syndrome but there are reports of less typical clinical cases with typical neuropathological changes including individuals with no demonstrable eye movement abnormalities. ${ }^{10-13}$ More recently five patients were reported with a clinical diagnosis of late onset dementia who shared some of the neuropathological features of PSP. ${ }^{14}$ These reports suggest that the clinical spectrum of PSP is wider than originally described, a development predicted by the original authors. ${ }^{1}$

PSP may also present neuropathologically in different, atypical forms. The most interesting and controversial aspect of the neuropathology of PSP is the involvement of the cerebral cortex. PSP was originally defined as a disease affecting the subcortical grey matter ${ }^{1}$ and the involvement of the neocortex has only more recently been recognised. Neocortical neurofibrillary tangles demonstrated by Bodian's silver impregnation and tau immunolabelling were found in PSP. These differed from the tangles of Alzheimer's disease both in their distribution ${ }^{15}$ and in their ultrastructure. ${ }^{16}$ In addition, neuropil threads, visualised by the silver impregnation technique of Gallyas and by tau antibody were found not only in the subcortical grey matter, ${ }^{17}$ but also in the neocortex and hippocampus. ${ }^{15}$ In our case although neocortical tangles were sparse, neuropil threads did occur. These neuropil threads or "curly" fibres, containing abnormal cytoskeletal components, could substantially contribute to the dementia. ${ }^{18}$

Our findings have two important implica- 
tions. Firstly, they show that PSP may affect the brain more extensively and more profoundly than previously envisaged: the pathological process may disrupt the neuronal cytoskeletal system more severely, resulting not only in tangles, but also in neuropil threads affecting both the subcortical grey matter and neocortex. Secondly, this hitherto unrecognised involvement of the neocortex must be a substantial factor in bringing about dementia, explaining some of the atypical cases presenting with late onset dementia, but without characteristic Alzheimer's disease pathology. From this it follows that the concept of PSP as a pure subcortical dementia should be reassessed.

There is some evidence in the literature for a familial form of PSP. Mata and colleagues ${ }^{19}$ reported a family of three siblings who developed a disease with dementia, kyphoscoliosis, pes cavus, rigidity, and pyramidal signs in their twenties. One member of this pedigree had a postmortem examination which showed many of the features of PSP (although the filaments were not examined ultrastructurally ${ }^{20}$ ). However many of the clinical manifestations in this family are not features of sporadic PSP. Ohara et al ${ }^{21}$ reported two siblings of a consanguineous marriage who developed a clinical syndrome similar to PSP. Necropsy in one patient revealed extensive neurofibrillary degeneration with prominent involvement of the limbic system. The neuropathological changes were atypical and the authors were unsure how to classify their disease. In addition to these two families one of the early series of PSP patients included a patient whose mother suffered from a similar illness. $^{22}$ In many of the reports of sporadic PSP it is unclear how carefully the family history was assessed.

There has been one case control study of PSP which examined the personal histories of 50 cases of PSP. and 100 age and sex matched controls. ${ }^{23}$ No definite familial cases were found but a family history of Parkinson's disease was 5.0-6.0 times commoner in affected patients than controls and a family history of Alzheimer's disease was 1.3-3.6 times commoner. These figures were not statistically significant and the authors explained the increase with higher awareness of these diseases in the families of patients with PSP. An alternative explanation is that these relatives had PSP, perhaps in atypical form, but received the diagnosis of Parkinson's disease or Alzheimer's disease. This occurred in our family. The late onset of symptoms may conceal the familial nature in some pedigrees due to unrelated morbidity and mortality before the age of onset. If the disease gene has low penetrance then a significant proportion of cases could be genetic.
The skilful technical assistance of Mrs Susan Wise and Mr A Chadwick is gratefully acknowledged. This work was partly supported by a special MRC project grant to the Alzheimer's Disease Brain Bank in the Department of Neuropathology, Institute of Psychiatry. Jeremy Brown is funded by the MRC We are grateful to Dr A Lees for his comments on the manuscript and to Dr S Singh for permission to include his patient in our study.

1 Steele JC, Richardson JC, Olszewski J. Progressive supranuclear palsy. A heterogeneous degeneration involving brainstem, basal ganglia and cerebellum with vertical gaze and pseudobulbar palsy, nuchal dystonia and dementia. Arch Neurol 1964;10:333-59.

2 Albert ML, Feldman RG, Willis AL. The subcortical dementia of progressive supranuclear palsy. $f$ Neurol dementia of progressive supranuclear

3 Maher ER, Smith EM, Lees AJ. Cognitive deficits in the Steele-Richardson-Olszewski syndrome (progressive supranuclear palsy). $f$ Neurol Neurosurg Psychiatry 1985;48:1234-9.

4 D'Antona R, Baron JC, Samson Y, et al. Subcortical dementia: frontal lobe hypometabolism detected by positron emmision tomography in patients with progressive supranuclear palsy. Brain 1985;108:785-99.

5 Leenders KI, Frackowiak RS, Lees AJ. SteeleRichardson-Olszewski syndrome. Brain energy metabolich blood flow and flo lism, blood flow and fluorodopa uptake measured by

positron emission tomography. Brain 1988;111:615-30.
Folstein MF, Folstein SE, McHugh PR. Mini Mental State: a practical method for grading the cognitive state State: a practical method for grading the cognitive state
of patients for the clinician. $\mathcal{F}$ Psychiat Res 1975;12: of patients

7 Lees AJ. The Steele-Richardson-Olszewski syndrome (progressive supranuclear palsy). In: Marsden CD Fahn S, eds. Movement disorders 2. London Butterworths, 1987; 272-87.

8 Maher ER, Lees AJ. The clinical features and natural history of Steele-Richardson-Olszewski syndrome (progressive supranuclear palsy). Neurology 1986;36:1005-8.

9 Collinge J, Brown J, Hardy J, et al. Inherited prion disease with $144 \mathrm{bp}$ gene insertion 2 . Clinical and pathological with 144 bp gene insertion 2. Clinice

10 Davis PH, Bergeron C, McLachlan DR. Atypical presentation of progressive supranuclear palsy. Ann Neurol 1985;17:337-43.

11 Jellinger K, Riederer $P$, Tomonaga M. Progressive supranuclear palsy: clinicopathological and biochemical studies. F Neurol Trans Suppl 1980;16:111-28.

12 Probst A. Dégénérescence neurofibrillaire sous-cortical sénile avec présence de tubules contournés et de filaments droits: forme atypique de la paralysie supranucléaire progressive. Rev Neurol (Paris) 1977;133:417-28.

13 Dubas F, Gray F, Escourolle R. Maladie de SteeleRichardson-Olszewski sans opthalmoplégie: six cas 407-16.

14 Masliah E, Hansen LA, Quijada $S$, et al. Late onset dementia with argyrophilic grains and subcortical tandementia with argyrophilic grains and subcortical tangles or atypical progre

15 Hauw J-J, Verny $M$, Delaère $P$, et al. Constant neurofibrillary changes in the neocortex in progressive supranuclear palsy. Basic differences with Alzheimer's disease and aging. Neurosci Lett 1990;119:182-6.

16 Takahashi $\mathrm{H}$, Oyanagi $\mathrm{K}$, Takeda $\mathrm{S}$, Hinokuma $\mathrm{K}$, Ikuta F. Occurrence of 15-nm wide straight tubules in the neocortical neurons in progressive supranuclear palsy. Acta Neuropathol 1989;79:233-9.

17 Probst A, Langui D, Lautenschlager C, et al. Progressive supranuclear palsy: extensive neuropil threads in addition to neurofibrillary tangles. Very similar antigenicity of subcortical neuronal pathology in progressive of subcortical neuronal pathology in progressive supranuclear palsy and

18 Braak H, Braak E, Grundke-Iqbal I, Iqbal K. Occurrence of neuropil threads in the senile human brain and in Alzheimer's disease: a third location of paired helical filaments outside of neurofibrillary tangles and neuritic plaques. Neurosci Lett 1986;65:351-5.

19 Mata $M$, Dorovini-Zis $K$, Wilson $M$, Young AB. New form of familial Parkinson-dementia syndrome: clinica and pathological findings. Neurology 1983;33:1439-43.

20 Ambrosetto P, Bacci A. New form of familial Parkinsondementia syndrome and progressive supranuclear palsy. Neurology 1984;34:1524.

21 Ohara S, Kondo $\mathrm{K}$, Morita $\mathrm{H}$, et al. Progressive supranuclear palsy-like syndrome in two siblings of a consanguineous marriage. Neurology 1992;42:1009-14.

22 David NJ, Mackey EA, Smith JL. Further observations in progressive supranuclear palsy. Neurology 1968;18: progress

23 Davis PH, Golbe LI, Duvoisin RC, Schoenberg BS. Risk factors for progressive supranuclear palsy. Neurology 1988;38:1546-52. 